\title{
Phase and Amplitude Regeneration through Sequential PSA and FWM Saturation in HNLF
}

\author{
K.R.H. Bottrill(1), F. Parmigiani( ${ }^{(1)}$, L. Jones ${ }^{(1)}$, G. Hesketh(1), D.J. Richardson ${ }^{(1)}$, P. Petropoulos ${ }^{(1)}$ \\ (1) Optoelectronics Research Centre, University of Southampton, SO17 1BJ, UK, krhb1g12@soton.ac.uk
}

\begin{abstract}
All-optical phase and amplitude regeneration of a QPSK signal using only two nonlinear stages is achieved by combining a PSA with a saturated pump-degenerate FWM based amplitude squeezer. EVM, phase noise and magnitude noise are all reduced by $50 \%$.
\end{abstract}

\section{Introduction}

Although phase regeneration alone may extend the reach of telecommunications signals, particularly phase shift keying (PSK) formats, it cannot be recursively applied to a signal ad infinitum; eventually the uncorrected amplitude noise will limit the signal to noise ratio of the transmission. Indeed, given that many phase sensitive amplifier (PSA) based phase regenerators result in an increase in amplitude noise, relatively few consecutive phase squeezing stages may be applied before they are no longer beneficial. Ultimately, to correct for non-deterministic noise, both the signal's phase and amplitude must be regenerated.

The few publications reporting PSA-based phase regeneration with simultaneous amplitude regeneration either make use of four wave mixing (FWM) saturation ${ }^{1}$, or operate the PSA within a nonlinear amplifying loop mirror (NALM) ${ }^{2}$. Despite successfully regenerating both phase and amplitude (and sometimes also offering multilevel functionality ${ }^{2}$ ), these schemes are often complicated in their optimisation and are limited in their regenerative ability either due to intrinsic noise processes, or as a result of a compromise between the underlying regenerative operations.

Instead of combining the two regenerative processes in one nonlinear stage, they may be applied sequentially in two independent processes ${ }^{3}$, allowing for easier optimisation but possibly requiring many stages of nonlinearity. In the investigation presented here, we combine two recent demonstrations of ours, the first, a compact, highly tunable scheme for phase regeneration of a quaternary PSK (QPSK) signal ${ }^{4}$ and the second, a saturated pump-degenerate FWM based amplitude squeezer, which makes use of a high pump to signal power ratio to reduce amplitude noise to phase noise conversion ${ }^{5}$. This enables the duties of phase and amplitude regeneration to be separated, greatly simplifying optimisation, all the while making use of only two nonlinear stages. As the phase regenerator is conjugating, self phase modulation (SPM) accrued by the signal in the phase regenerator is undone as it propagates through the amplitude regenerator. We analyse the regenerator in terms of its effect on the signal's noise statistics, its constellation plots as well as providing BER measurements, for various combinations of broadband phase noise and amplified spontaneous emission (ASE).

\section{Concept}

The scheme consists of two simple nonlinear processing stages. The first is a wavelength converting, idler-free PSA which squeezes the signal phase by simultaneously producing the signal's 3rd phase harmonic using a cascaded signaldegenerate FWM process and coherently adding to it the signal's phase conjugate using a copropagating dual-pumped FWM process. This scheme is notable not only for achieving phase squeezing using a single nonlinear stage, but also its ease of tunability and flexible operating power, advantages paid for through its wavelength converting nature - the regenerated signal is produced at the location of the signal's 3rd harmonic and is conjugated ${ }^{4}$.

The role of the second nonlinear stage is fourfold: 1) conversion of the signal back to its original wavelength; 2) restoration of the signal to its unconjugated state; 3 ) regeneration of the signal's amplitude; 4) compensation of the SPM induced by the first nonlinear stage. All of these goals can be readily achieved by employing a pumpdegenerate parametric amplifier operated in saturation and selecting the conjugated idler. As shown in ${ }^{5}$, saturation of the signal gain in such a scheme may be achieved for a large range of pump to signal power ratios; the larger the ratio, the smaller the SPM induced in the signal. By controlling this ratio, we may balance the SPM induced by the amplitude regenerator with that in- 


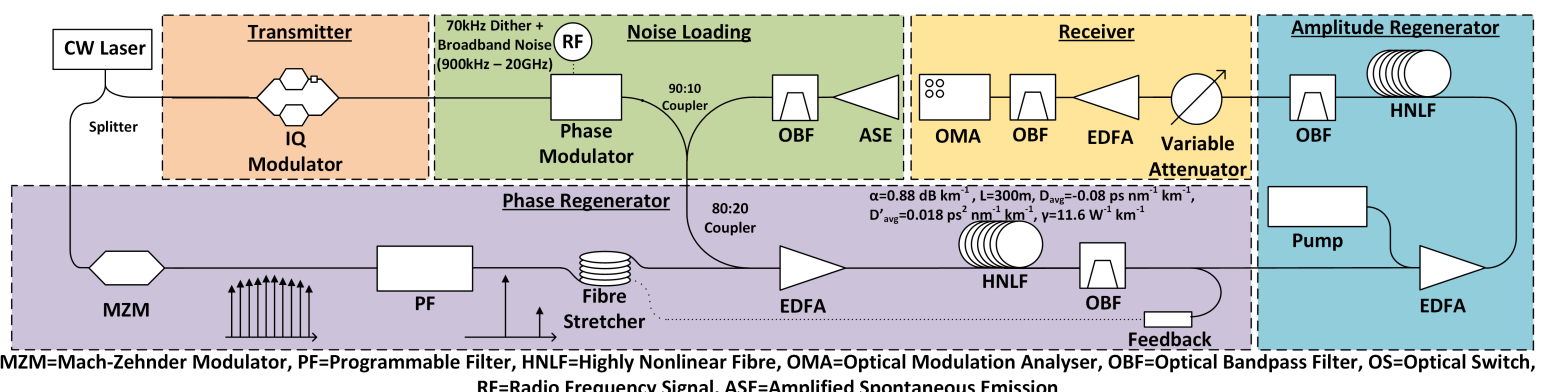

Fig. 1: Experimental setup of the regenerator showing the two underlying phase and amplitude regenerative stages.

duced by the phase regenerator.

\section{Experimental Setup}

Figure 1 shows the experimental setup of the regenerator, with the various stages colour-coded for clarity. Firstly, light from a laser operating at 192.5 THz is divided into two paths. One leads to the transmitter stage, where a 10 Gbaud QPSK modulation is applied. The modulated signal then enters the noise loading stage, where a $70 \mathrm{kHz}$ sinusoidal phase dither and broadband phase noise are added using a phase modulator, and inband ASE is added using an EDFA, a filter and a coupler. Meanwhile, the other path of the laser is used to produce an optical frequency comb with $40 \mathrm{GHz}$ spacing using a Mach-Zehnder modulator. This comb is then passed through a programmble filter, where the undesired frequencies are filtered out, leaving only lines at $192.54 \mathrm{THz}$ (P1) and 192.38 THz (P2) which are to be used as the pumps in the PSA stage.

The signal and pumps are then recombined and amplified to a total power of about $25 \mathrm{dBm}$ (spectrum provided in Fig. 2-a) before entering $300 \mathrm{~m}$ of low dispersion HNLF to undergo PSA, resulting in the output presented in Fig. 2-b. As described above, the PSA stage is wavelength converting, and so to extract the phase squeezed signal, a filter is placed at $192.42 \mathrm{THz}$, the location of the signal's third harmonic.

The phase squeezed, conjugated and wavelength converted signal then enters the amplitude squeezing stage. All that is required is for a single CW pump at $192.46 \mathrm{THz}$ to be coupled with the signal and for the pair to be amplified to approximately $35 \mathrm{dBm}$ before being launched into multiple segments of low dispersion HNLF (totalling $2 \mathrm{~km}$ in length) connected with optical isolators to increase the Brillouin threshold ${ }^{5}$. The input and output spectra of the HNLF are given in Fig. 2-c and Fig. 2-d, respectively. At the end of the HNLF, a filter is used to extract the output, which is unconjugated relative to the original signal and located at the original wavelength $(192.5 \mathrm{THz})$. The system was tuned at each stage by analysing the output using an OMA and optimising to minimise phase noise at the very end of the regenerator.

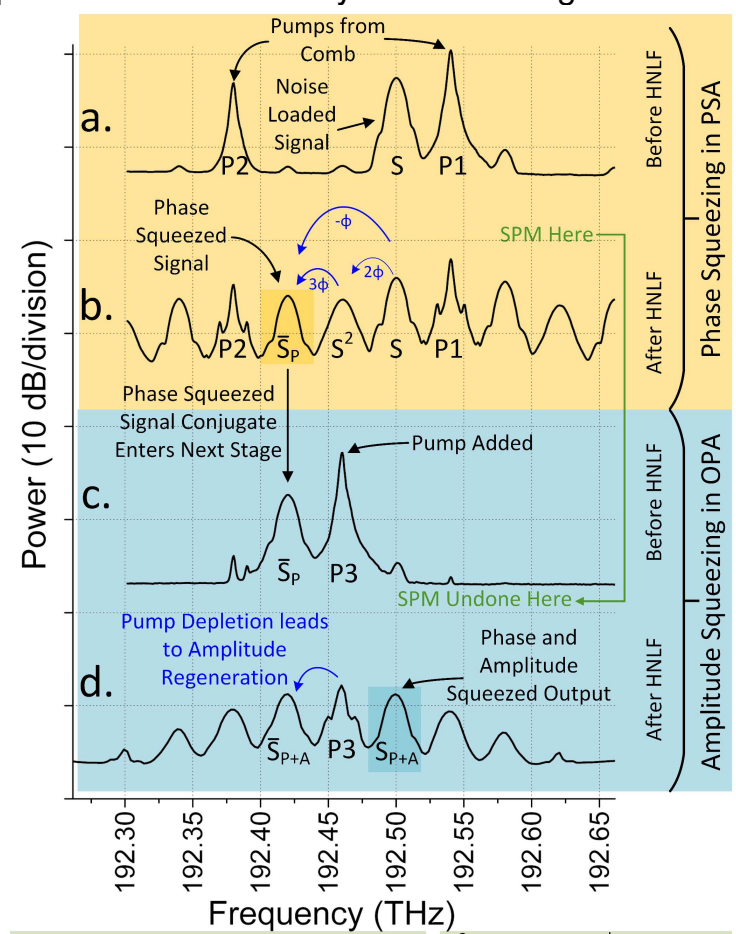

$\bar{S}_{p}$ : Phase Squeezed Signal Conjugate $S^{2}$ : Signal's $2^{\text {nd }}$ Harmonic $S_{P+A}$ : Phase and Amplitude Squeezed Signal S: Input Signal $\bar{S}_{p+A}:$ Phase and Amplitude Squeezed Signal Conjugate

Fig. 2: Spectra: a. Before PSA; b. After PSA; c. Before amplitude regeneration; $d$. After amplitude regeneration.

\begin{tabular}{|c|c|c|c|c|c|}
\hline Noise Scenario & Metric & Units & $\begin{array}{l}\text { Before } \\
\text { Regen }\end{array}$ & $\begin{array}{l}\text { Phase } \\
\text { Regen }\end{array}$ & $\begin{array}{c}\text { Phase + } \\
\text { Amp } \\
\text { Regen }\end{array}$ \\
\hline \multirow{3}{*}{ No Added Noise } & EVM & $\% \mathrm{rms}$ & 7.3 & 10.4 & 7.3 \\
\hline & $\Delta \phi$ & deg rms & 3.0 & 3.8 & 3.4 \\
\hline & $\Delta M a g$ & $\% \mathrm{rms}$ & 5.1 & 8.0 & 4.3 \\
\hline \multirow{3}{*}{ Phase Noise Only } & EVM & $\%$ rms & 14.7 & 11.3 & 8.2 \\
\hline & $\Delta \phi$ & deg $\mathrm{rms}$ & 7.9 & 4.1 & 3.9 \\
\hline & $\Delta M a g$ & $\%$ rms & 5.3 & 8.8 & 4.5 \\
\hline \multirow{3}{*}{ Phase Noise + ASE } & EVM & $\% \mathrm{rms}$ & 21.9 & 17.7 & 11.7 \\
\hline & $\Delta \phi$ & deg rms & 10.2 & 6.3 & 5.4 \\
\hline & $\Delta M a g$ & $\%$ rms & 13.1 & 16.0 & 7.3 \\
\hline
\end{tabular}

Table 1: Table of noise statistics of the signal.

\section{Results}

For varying input noise scenarios and at different stages in the scheme, constellation plots and BER curves are provided in Figs. 3 -a and -b, respectively, with noise statistics provided in Table 1. Without additional noise being added to the signal, the constellation plot after phase and am- 
plitude regeneration (blue plot Fig. 3-a, top row) is comparable to that before regeneration (yellow plot 3-a) and shows a small decrease in amplitude noise, but a small increase in phase noise, with a marginal power penalty of $0.23 \mathrm{~dB}$ for a BER of $10^{-4}$, as shown in Fig. 3-b (black squares).

The second row of plots in Fig. 3-a shows the case phase noise only was added resulting in a signal phase noise of $8 \mathrm{deg} \mathrm{rms}$. After the phase regenerator (green), the phase noise has been greatly reduced, but at the expense of an increase in amplitude noise, which is typical behaviour for this regenerator ${ }^{1,4}$. Comparison of the constellation plots after phase regeneration (green) and after both phase and amplitude regeneration (blue), clearly shows the benefit of this additional, amplitude regenerating stage. This is also reflected in the noise statistics in Table 1 and it is interesting to note how the phase noise after amplitude regeneration is smaller than that before; this is the effect of the optical phase conjugation discussed earlier. The BERs for this noise level (blue circles) show an improvement in receiver sensitivity of about $1.1 \mathrm{~dB}$ for a BER of $10^{-4}$.

The third row in Fig. 3-a is the result of contaminating the phase noise loaded signal with ASE. Once again, the regenerator clearly reduces the noise, and the amplitude regenerator proves its worth in reducing the large amount of amplitude noise present after phase squeezing as well as undoing the SPM accrued in the PSA. For this case (orange triangles, Fig. 3-b), the improvement in receiver sensitivity is $2 \mathrm{~dB}$ for a BER of $10^{-4}$ and we witness an extra 1 deg rms reduction in phase noise through the addition of the amplitude regenerator. The error floor in the BER curves is a consequence of the noise loading that takes place after the transmitter. After the phase and amplitude regenerating scheme, the EVM is roughly halved for this noise level (see Table 1).

\section{Conclusion}

Phase and amplitude squeezing was demonstrated using an idler-free PSA followed by a saturated pump-degenerate FWM based wavelength converter. By exploiting phase conjugation in the PSA, SPM accrued in the first nonlinear stage may be undone in the second, reducing output phase noise and permitting higher launch powers to be used. A near halving of EVM, phase noise and magnitude noise is demonstrated using the scheme, and BER measurements demonstrate an improvement in receiver sensitivity of $2 \mathrm{~dB}$ for a BER of $10^{-4}$ in the highest noise case.

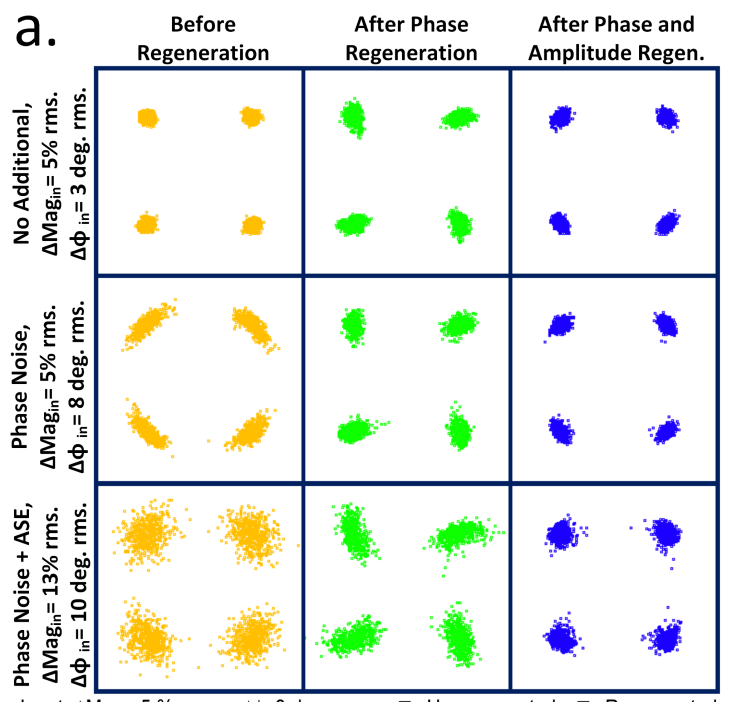

Input: $\Delta$ Mag. $=5 \%$ rms. $\Delta \phi=3$ deg. rms. 口 Unregenerated D Regenerate Input: $\Delta$ Mag. $=5 \%$ rms. $\Delta \phi=8$ deg. rms. 0 Unregenerated $\bullet$ Regenerated Input: $\Delta$ Mag. $=13 \%$ rms. $\Delta \phi=10$ deg. rms. $\Delta$ Unregenerated $\Delta$ Regenerated

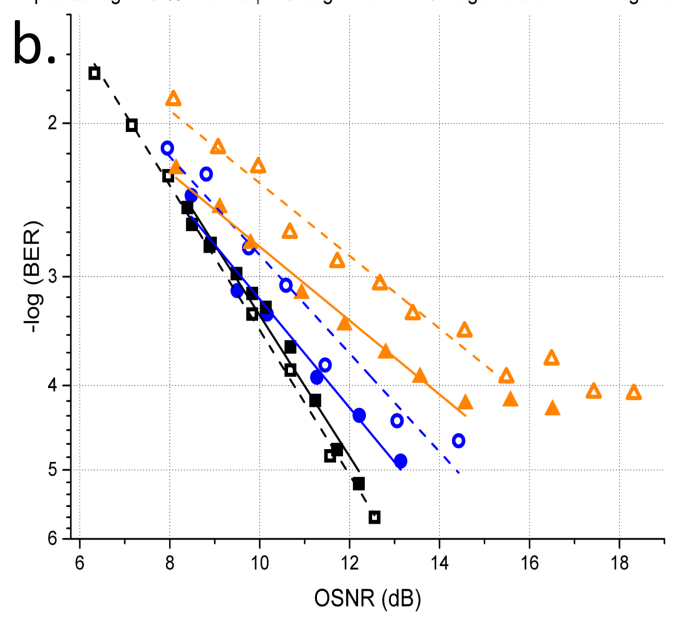

Fig. 3: a. Constellation plots; b. BER curves.

\section{Acknowledgements}

This research is supported by the EPSRC grant EP/I01196X. Dr F. Parmigiani is a Royal Academy of Engineering/EPSRC Research Fellow.

\section{References}

[1] Kakande, J. et al., "QPSK phase and amplitude regeneration at 56 Gbaud in a novel idler-free non-degenerate phase sensitive amplifier," OFC/NFOEC 2011, Paper OMT.4.

[2] T. Roethlingshoefer et al., "Cascaded phase-preserving multilevel amplitude regeneration," Opt. Express 22, 31729-31734 (2014).

[3] Mohajerin-Ariaei, A. et al., "Bit-rate-tunable regeneration of 30-Gbaud QPSK data using phase quantization and amplitude saturation," ECOC 2014, Paper P.3.20.

[4] K. Bottrill et al., "FWM-based, Idler-free Phase Quantiser with Flexible Operating Power," OFC 2015, Paper W4C.3.

[5] K.R.H. Bottrill et al., "Investigation into the Role of Pump to Signal Power Ratio in FWM-based Phase Preserving Amplitude Regeneration", CLEO 2015, Paper SM2M.1. 